\title{
Encryption of 3D Point Cloud Object with Deformed Fringe
}

\author{
Xin Yang ${ }^{1}$ and Hongbo Zhang ${ }^{2}$ \\ ${ }^{1}$ Institute of Information Optics Engineering, Soochow University, Suzhou, Jiangsu 210056, China \\ ${ }^{2}$ Bradley Department of Electrical and Computer Engineering, Virginia Tech, Blacksburg, VA 24060, USA
}

Correspondence should be addressed to Xin Yang; 2006endeavour@163.com

Received 17 October 2015; Revised 11 January 2016; Accepted 14 January 2016

Academic Editor: Paramasivam Senthilkumaran

Copyright (C) $2016 \mathrm{X}$. Yang and H. Zhang. This is an open access article distributed under the Creative Commons Attribution License, which permits unrestricted use, distribution, and reproduction in any medium, provided the original work is properly cited.

\begin{abstract}
A 3D point cloud object encryption method was proposed with this study. With the method, a mapping relationship between 3D coordinates was formulated and $Z$ coordinate was transformed to deformed fringe by a phase coding method. The deformed fringe and gray image were used for encryption and decryption with simulated off-axis digital Fresnel hologram. Results indicated that the proposed method is able to accurately decrypt the coordinates and gray image of the $3 \mathrm{D}$ object. The method is also robust against occlusion attacks.
\end{abstract}

\section{Introduction}

Image encryption has been extensively studied in the past. It includes encryption of $2 \mathrm{D}$ and $3 \mathrm{D}$ objects through computational methods. For example, discrete fractional Fourier transform, Fibonacci transformation, and discrete cosine transform have been used to for image or 3D object encryption [1-3]. Alternatively, holographic encryption has also been applied for encrypting and decrypting information. Holographic encryption has advantages in providing flexibility in selection of various encryption parameters including optical wavelength, diffraction distance, and polarization. The encryption of 2D image uses optical methods such as wavelength multiplexing method $[4,5]$, two-step phaseshifting interferometry [6], computer-generated hologram [7], joint transform correlator [8], and modulation of plane wave [9] as reference wave. The significance of 3D information encryption is also obvious and many methods for the encryption of 3D objects such as using digital holography [10-12] and computer-generated [13] have been proposed. However, for most of 3D object encryption, object information has yet been fully reconstructed in terms of accuracy and robustness, although the $3 \mathrm{D}$ information is very important for practical utilization $[14,15]$. It is mostly due to the complexity of the computation and sensitivity of the parameters inherited in the methods.
More robust methods were created to reduce the sensitivity of the encryption parameters hence to increase the validity of the encryption under different environment. Among them, the integral imaging method has been popularized due to its use of structured pinholes to enhance the data redundancy and encryption robustness, yet the method involves use of complex experimental setup and simulation $[1,16]$. Advanced 3D digital holography that has been also applied for 3D image encryption, though robust, lacks accuracy and is insufficiently strong in encryption process due to the direct use of object coordinate information in the encryption process [17].

In this paper, we proposed robust and accurate, yet less computation intensive 3D point cloud object encryption. At the center of the method, a mapping relationship between $X, Y$, and $Z$ was formulated. $Z$ coordinate of the $3 \mathrm{D}$ object was phase coded and subsequently transformed to deformed fringe. With deformed fringe and gray image of the 3D object, a conventional off-axis digital Fresnel holography method was utilized to encrypt and record their information. Following the decryption and reconstruction, both deformed fringe and gray image were reconstructed. 3D object was hence reconstructed with $X, Y, Z$, and gray image information.

\section{Methods}

The angular orthogonal projection geometry [18] is used to convert the $3 \mathrm{D}$ point objects of one specific perspective 
into $x-y$ plane and $Z$ coordinate. Figure 1 is the angular orthogonal projection and the projection image coordinate $\left(x_{i}, y_{i}\right)$ and the object point $\left(x_{k}, y_{k}, z_{k}\right.$, gray $\left._{k}\right)$ from a gray model are related by

$$
\begin{aligned}
& x_{i}=x_{k} \cos (\varphi)-z_{k} \sin (\varphi), \\
& y_{i}=y_{k} \cos (\theta)-z_{k} \sin (\theta) \cos (\varphi)-x_{k} \sin (\varphi) \sin (\theta) .
\end{aligned}
$$

For a specific situation when $\varphi=\pi / 2$ and $\theta=\pi / 2$,

$$
\begin{aligned}
& x_{i}=x_{k}, \\
& y_{i}=y_{k} .
\end{aligned}
$$

A little difference from the traditional angular orthogonal projection is that two arrays gray and $Z$ are used to save the gray value and $z$ coordinates. The sampling intervals in the projection plane are $\Delta x$ and $\Delta y$, respectively, in $x$ and $y$ coordinate and the resolution of projection plane is $M$ by $N$. The arrays are calculated based on the following rules:

$$
\begin{gathered}
Z(i, j)=Z\left(\frac{y_{k}}{\Delta y}+\frac{M}{2}, \frac{x_{k}}{\Delta x}+\frac{N}{2}\right)=z_{k}, \\
\operatorname{Gray}(i, j)=\operatorname{Gray}\left(\frac{y_{k}}{\Delta y}+\frac{M}{2}, \frac{x_{k}}{\Delta x}+\frac{N}{2}\right)=\text { gray }_{k} .
\end{gathered}
$$

A $3 \mathrm{D}$ point cloud object was used. The size of this model is $21.25 \times 34.87 \times 17.89 \mathrm{~mm}^{3}$. The $k$ th point of the $3 \mathrm{D}$ object can be represented by $P_{k}\left(x_{k}, y_{k}, z_{k}\right.$, gray $)$, where $k$ is within the range of 1 to $K . K$ is the number of object points, where $\Delta x$ and $\Delta y$ are both $0.02 \mathrm{~mm}$, which gives the resolution of 300 $\times 300$ corresponding to $M$ rows multiplied by $N$ columns in $x-y$ plane for the point cloud object as is shown in Figure 2.

The calculation of $x$ and $y$ coordinates can be extracted from (3), which can be expressed as

$$
\begin{aligned}
& x_{k}(i, j)=\left(j-\frac{N}{2}\right) \Delta x, \\
& y_{k}(i, j)=\left(i-\frac{M}{2}\right) \Delta y .
\end{aligned}
$$

Steps of proposed method for encryption and decryption of the $3 \mathrm{D}$ object are shown in Figure 3. below.

The detailed description of the two steps is presented in

Step 1 (transformation of $Z$ coordinate to deformed fringe). The method starts from decomposing the 3D object into $x-y$ plane and $Z$ coordinate for a given view port. It is followed by transforming $Z$ coordinate of the $3 \mathrm{D}$ object into phase $\phi(i, j)$ shown below:

$$
\phi(i, j)=\xi\left[2 \pi \frac{Z(i, j)-M_{i}}{M_{x}-M_{i}}-\pi\right],
$$

where $M_{x}=\max \{Z\}, M_{i}=\min \{Z\}$, and $\xi$ is a constant between 0 and $1 . \max \{Z\}$ is the calculation of maximum value of $Z$ and $\min \{Z\}$ is the calculation of minimum value of $Z$.

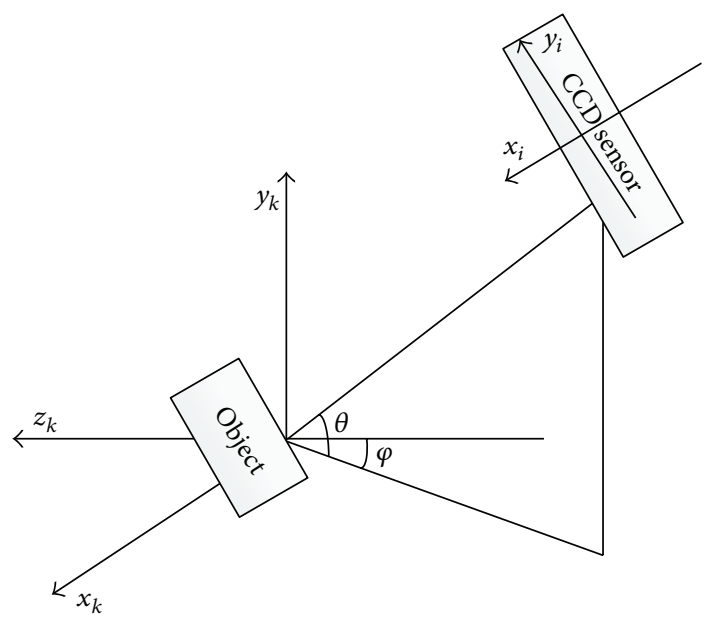

FIGURE 1: The angular orthogonal projection geometry.

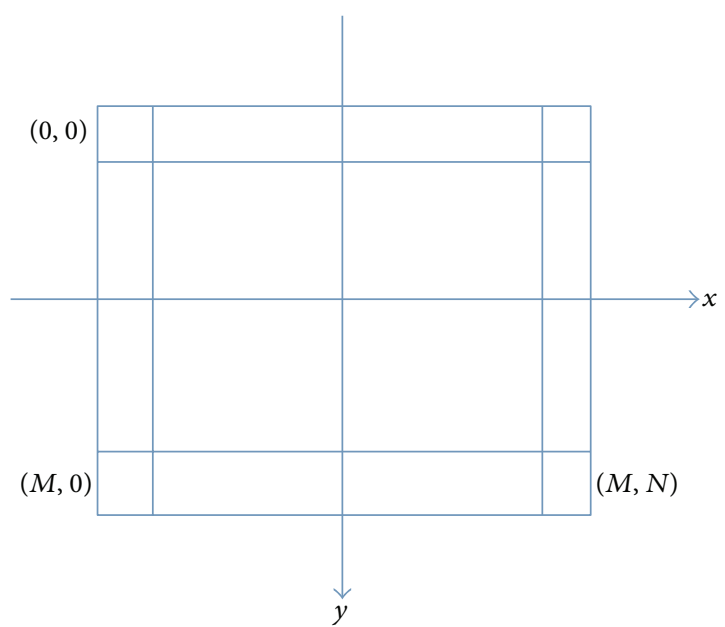

FIGURE 2: The coordinate system of 3D point cloud in $x-y$ plane.

With a proper selection of $\xi$, the range of $\phi$ could be within $[-\pi, \pi] . M_{x}=1.7893, M_{i}=0$, and $\xi=0.9$ was used.

The phase is further coded to deformed fringe with phase coding method inspired by Fourier transform profilometry $[19,20]$, which can be expressed as

$$
\begin{aligned}
I(x, y)= & a+b \cos \left(2 \pi f_{x} x+\phi\right) \\
= & a+\frac{b}{2} \exp \left[i\left(2 \pi f_{x} x+\phi\right)\right] \\
& +\frac{b}{2} \exp \left[-i\left(2 \pi f_{x} x+\phi\right)\right],
\end{aligned}
$$

where $a$ and $b$ are some constants and $f_{x}$ carrier frequency is 0.11 .

$Z$ coordinate and deformed fringe are shown in Figure 4. Figure $4(\mathrm{a})$ is the normalized $Z$ coordinate of $3 \mathrm{D}$ object. Figure $4(\mathrm{~b})$ is the deformed fringe coded from phase calculated from the mapping formula between height and phase. Figure $4(\mathrm{c})$ is the gray image of $3 \mathrm{D}$ object. 


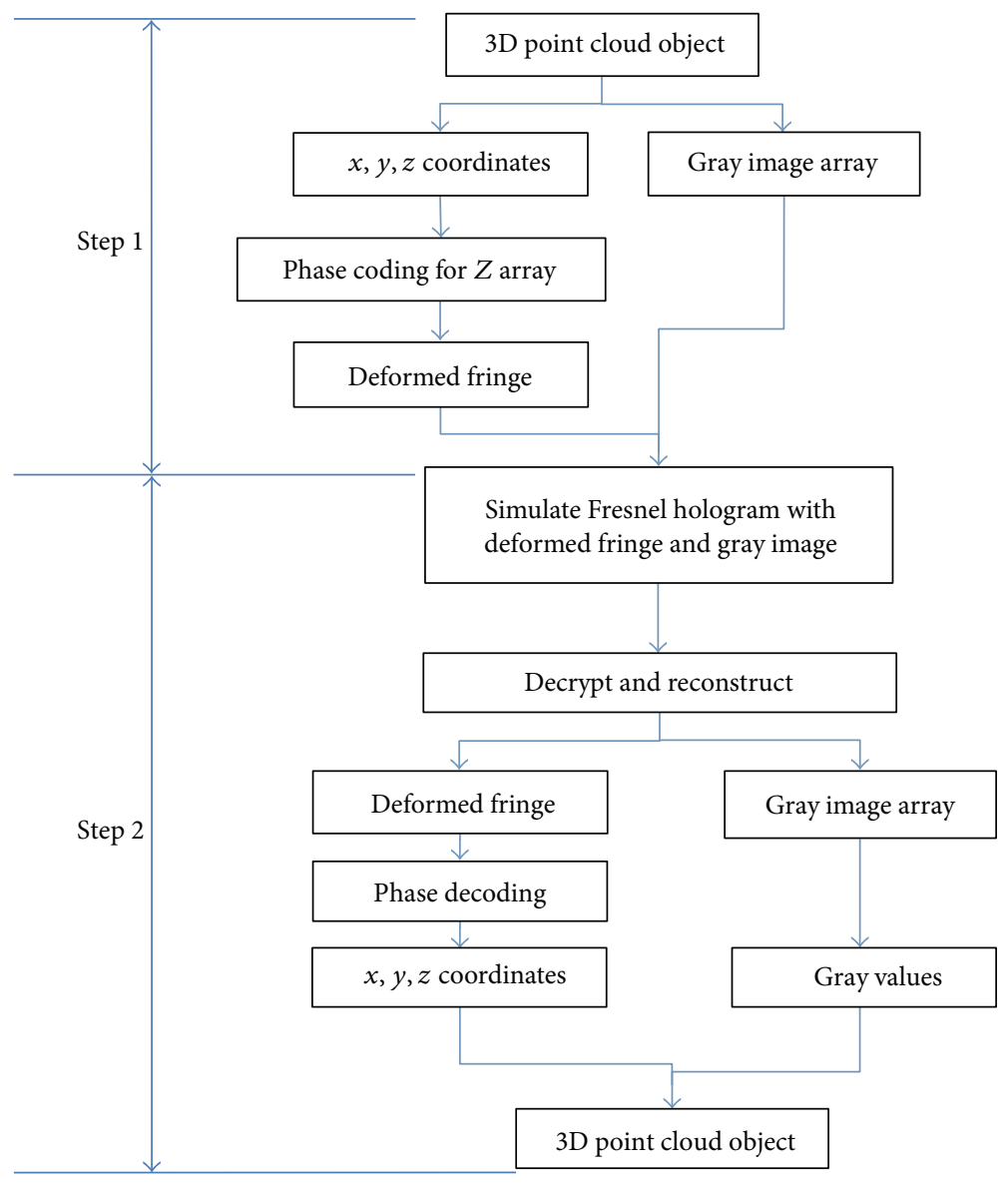

FIGURE 3: Steps of encryption and decryption for 3D point cloud object.

Step 2 (encryption and decryption). Multiplexing methods $[4,5]$ are often used in information encryption with Fresnel hologram. The space multiplexing method, the combination of deformed fringe and gray image as object, is used to simulate off-axis Fresnel holography shown in Figure 5. In the simulation, $4 f$ optical system constructed from lenses $L_{1}$ and $L_{2}$ was used. Laser with $\lambda=632.8 \mu \mathrm{m}$ was used in the simulation. Two random phase masks, which are also used as encryption keys, $\mathrm{PR}_{1}$ and $\mathrm{PR}_{2}$, were used to encrypt the object and reference waves. The object wave on the hologram plane is described:

$$
\begin{aligned}
O & \left(x_{h}, y_{h}\right) \\
& =\text { Fresnel }\left\{\operatorname{obj}(x, y) \exp \left[i \phi_{\mathrm{PR}_{1}}(x, y)\right], z_{f}\right\},
\end{aligned}
$$

where Fresnel $\left\{\bullet, z_{f}\right\}$ is the Fresnel diffraction in (8) of • with a diffraction distance $z_{f}$. $z_{f}=600 \mathrm{~mm}$. obj $(x, y)$ is the distribution of object and $\phi_{\mathrm{PR}_{1}}(x, y)$ is the phase due to random phase mask $\mathrm{PR}_{1}$. Consider

$$
\begin{gathered}
\text { Fresnel }\left\{\bullet, z_{f}\right\}=\frac{1}{i \lambda z_{f}} \exp \left(z_{f}\right) \\
\cdot \exp \left[i \frac{k}{2 z_{f}}\left(x_{h}{ }^{2}+y_{h}{ }^{2}\right)\right]
\end{gathered}
$$

$$
\begin{aligned}
& \iint \cdot \exp \left[i \frac{k}{2 z_{f}}\left(x^{2}+y^{2}\right)\right] \\
& \cdot \exp \left[-j \frac{2 \pi}{\lambda z_{f}}\left(x_{h} x+y_{h} y\right)\right] d x d y .
\end{aligned}
$$

The Fresnel hologram is written as

$$
\begin{aligned}
H\left(x_{h}, y_{h}\right)= & \left|O\left(x_{h}, y_{h}\right)\right|^{2}+\left|R\left(x_{h}, y_{h}\right)\right|^{2} \\
& +O\left(x_{h}, y_{h}\right) R^{*}\left(x_{h}, y_{h}\right) \\
& +O^{*}\left(x_{h}, y_{h}\right) R\left(x_{h}, y_{h}\right),
\end{aligned}
$$

where $R\left(x_{h}, y_{h}\right)=\exp \left[i \phi_{\mathrm{PR}_{1}}(x, y)\right]$ and asterisk denotes the conjugate wave of $O\left(x_{h}, y_{h}\right)$ and $R\left(x_{h}, y_{h}\right)$. It consists of DCs (first two components), first order, and conjugate terms (third and fourth items). The resolution of hologram is $1400 \times 1400$ and the sampling interval in hologram plane is $10 \mu \mathrm{m}$ in both $x$ and $y$ directions. The angle between the reference and object waves is 2.4 degrees. 


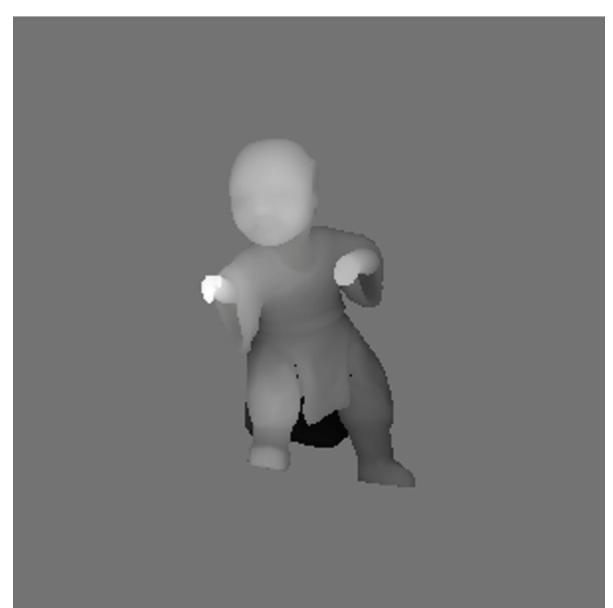

(a)

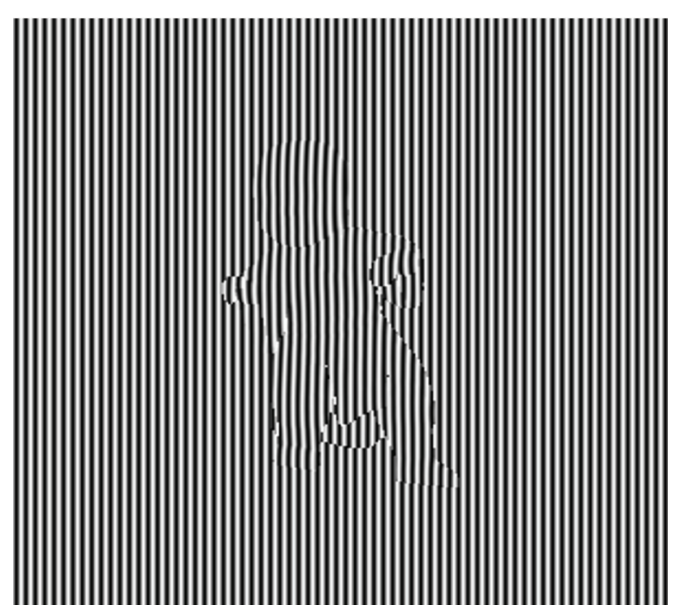

(b)

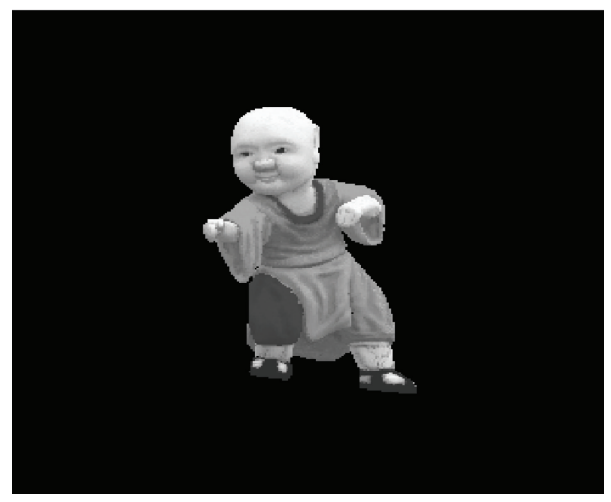

(c)

FIGURE 4: Normalized $Z$ coordinate of 3D object (a), deformed fringe coded from phase (b), and gray image (c).

The hologram reconstruction and decryption process is written as

$$
\begin{aligned}
& \operatorname{obj}(x, y) \\
& \quad=\text { Fresnel }\left\{O\left(x_{h}, y_{h}\right) R^{*}\left(x_{h}, y_{h}\right) R\left(x_{h}, y_{h}\right),-z_{f}\right\} \\
& \quad \cdot \exp \left[-i \phi_{\mathrm{PR}_{2}}(x, y)\right]
\end{aligned}
$$

where $\phi_{\mathrm{PR}_{2}}(x, y)$ is the phase due to random phase mask $\mathrm{PR}_{2}$.

The phase masks $\mathrm{PR}_{1}$ and $\mathrm{PR}_{2}$ are generated based on logistic chaos sequence $[20,21]$, which is expressed as

$$
X_{j+1}=\mu X_{j}\left(1-X_{j}\right)
$$

where $0 \leq \mu \leq 4$ and $X_{j} \in(0,1)$. $\mu$ is within the range of $[3.5999456,4]$ such that $X$ becomes chaotic to increase the encryption strength. For two random phase masks, the values of $\mu^{1}=3.625875, X_{1}{ }^{1}=0.6$ and $\mu^{2}=3.625875, X_{1}{ }^{2}=0.5632$ were used.

Following the hologram reconstruction, gray image and deformed fringe are obtained. For deformed fringe, with a specific choice of carrier frequency $f_{x}$ shown in (6) and

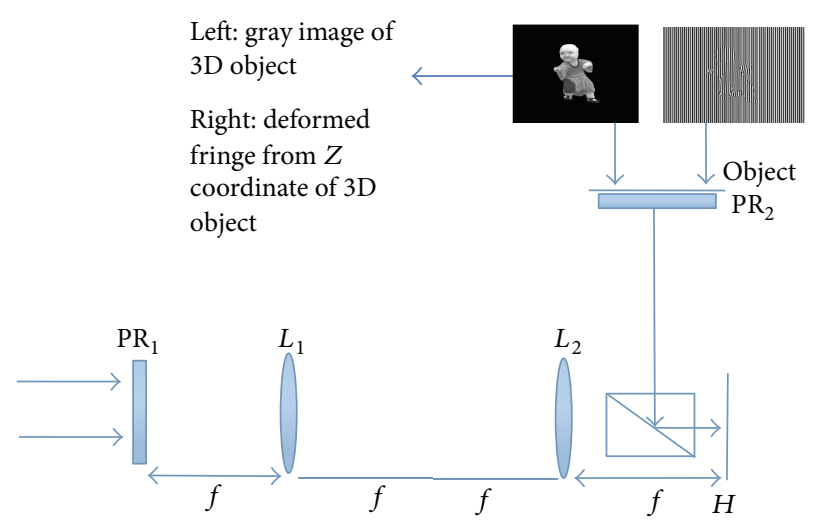

FIGURe 5: A $4 f$ optical holographic encryption system. $\mathrm{PR}_{1}$ and $\mathrm{PR}_{2}$ are encryption keys.

filtering in frequency domain, the deformed fringe can be simplified to $[16,17]$

$$
I_{a}(x, y)=\frac{b}{2} \exp (i \phi)
$$




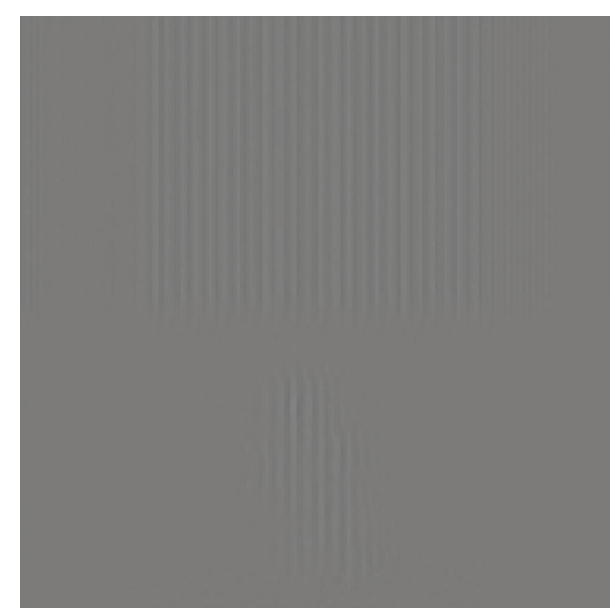

(a)

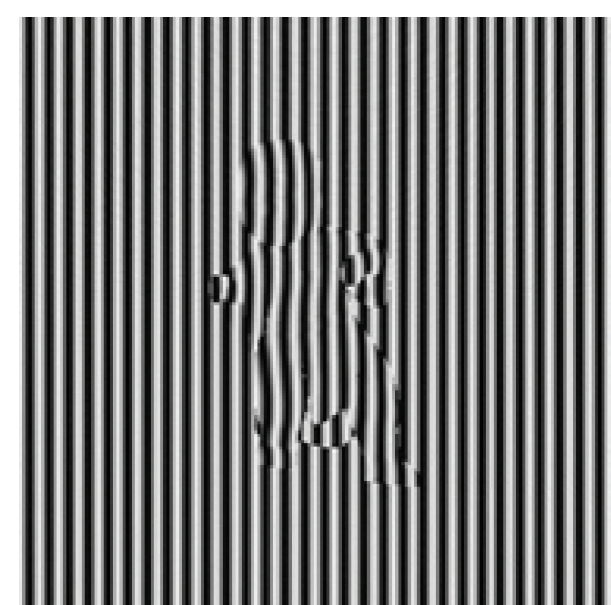

(b)

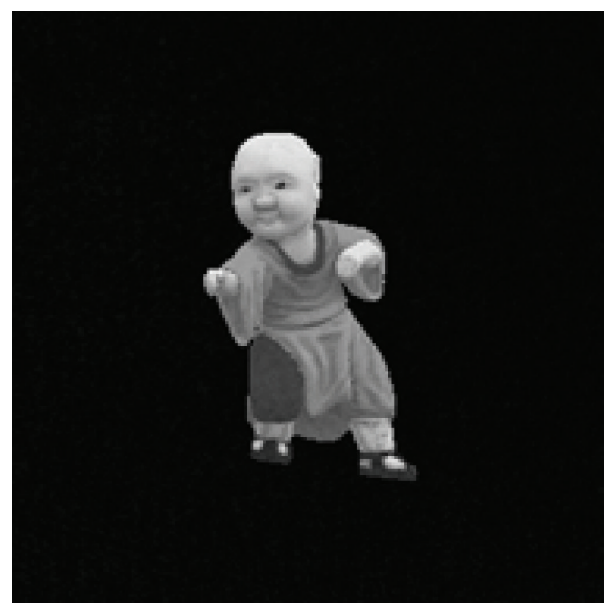

(c)

FIGURE 6: Encrypted Fresnel hologram (a), reconstructed deformed fringe (b), and gray image (c).

The phase $\phi(i, j)$ can be calculated based on simplified deformed fringe:

$$
\phi(i, j)=\operatorname{atan}\left[\frac{\operatorname{real}\left\{I_{a}\right\}}{\operatorname{imag}\left\{I_{a}\right\}}\right] .
$$

$\operatorname{real}\left\{I_{a}\right\}$ is the real part of $I_{a}$ and $\operatorname{imag}\left\{I_{a}\right\}$ is the imaginary part of $I_{a}$. The phase was truncated within the range of $[-\pi, \pi]$ avoiding the phase unwrap problem $[16,17]$.

With phase $\phi(i, j), Z$ coordinate can be reconstructed based on (5). Using reconstructed gray image and $Z$ coordinate and sampling intervals $\Delta x$ and $\Delta y$ in $x$ and $y$ directions, the $x$ and $y$ coordinates of $3 \mathrm{D}$ object can also be reconstructed using (4).

Simulated Occlusion Attacks. The occlusion attacks of the Fresnel hologram simulation were conducted. It was done through removal of partial Fresnel hologram data. The occlusion ratio is defined as the ratio of the removed Fresnel hologram data dividing the total Fresnel hologram data. The mean square error (MSE) between real $z$ coordinates and reconstructed $z$ coordinates was calculated and correlation coefficient (CC) of reconstructed gray image comparing with original gray image was also tested when occlusion ratio was different.

Direct $Z$ Coordinate versus Deformed Fringe Encryption. In order to comparably quantify the robustness of using deformed fringe for $Z$ coordinate encryption, comparison of performance of direct (just using) $Z$ coordinate versus deformed fringe encryption was conducted. It was used to illustrate the relationship between occlusion ratio and mean square error of $Z$ coordinate reconstruction by just using $Z$ coordinate versus using deformed fringe for Fresnel hologram encryption.

\section{Results}

The encrypted Fresnel hologram and reconstructed deformed fringe and gray image of 3D object are shown in Figure 6. Correlation coefficient between the reconstructed gray image and original gray image is 0.9824 . 


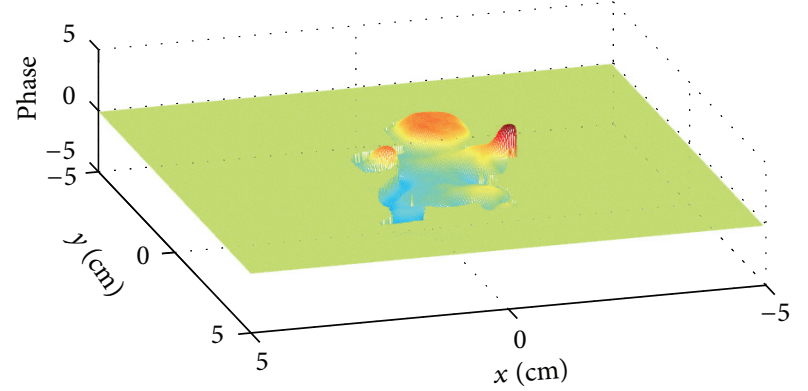

(a)

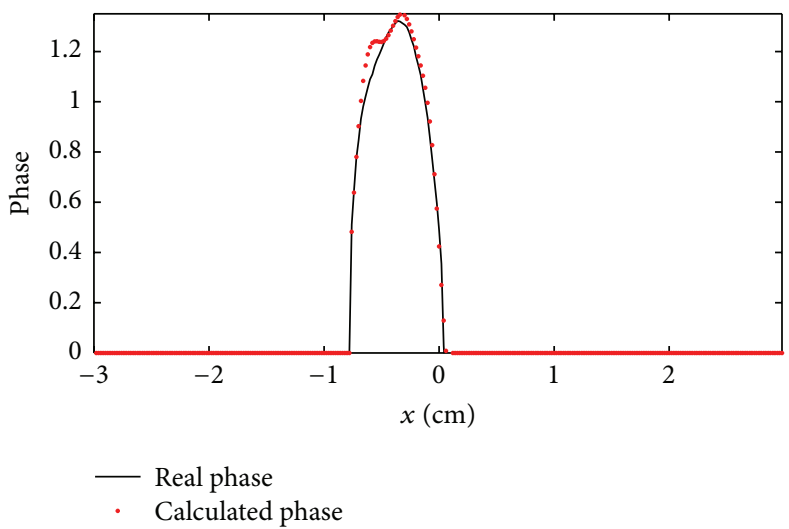

(c)

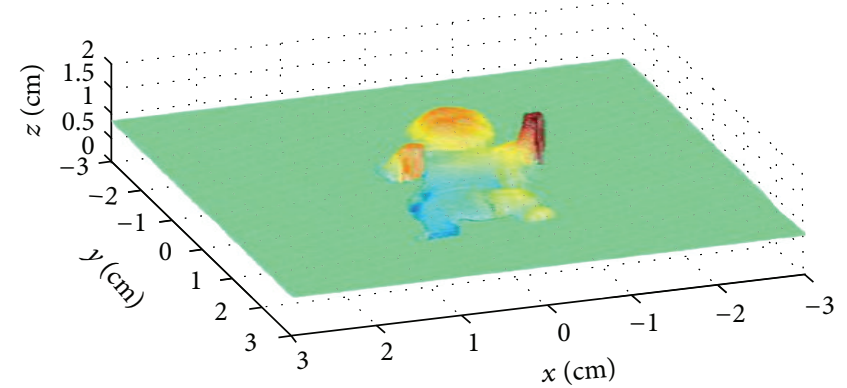

(b)

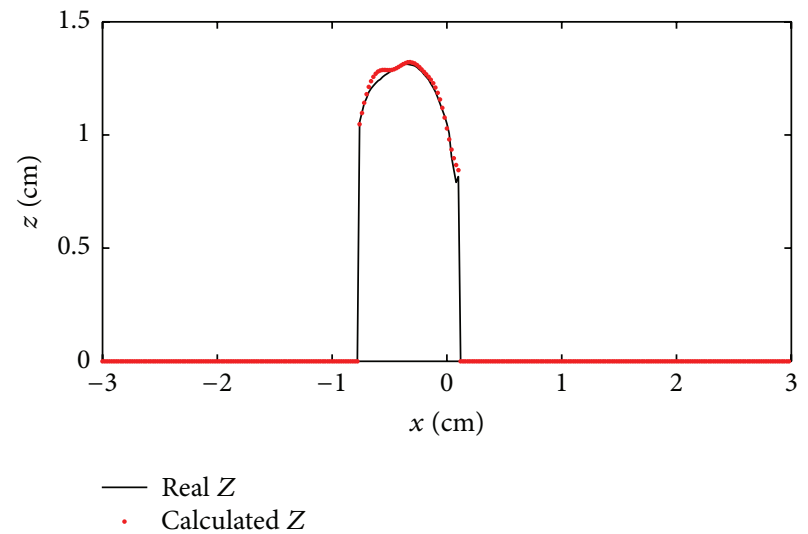

(d)

Figure 7: Reconstructed phase (a) and $Z$ coordinate (b), comparison between original and reconstructed phase (c) and $Z$ (d).

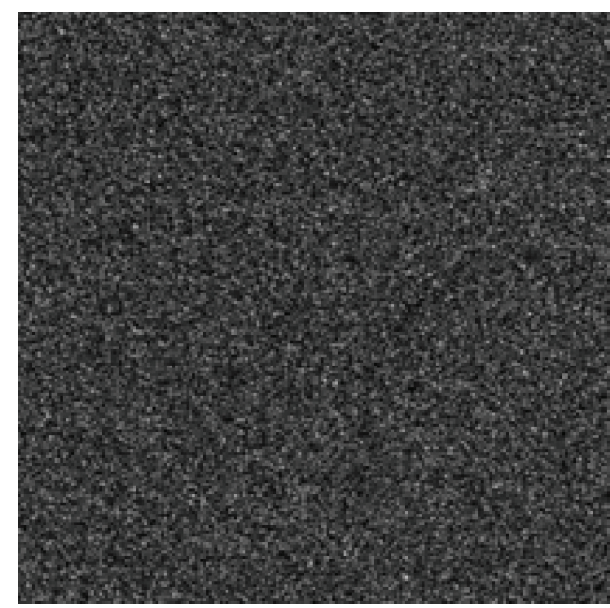

(a)

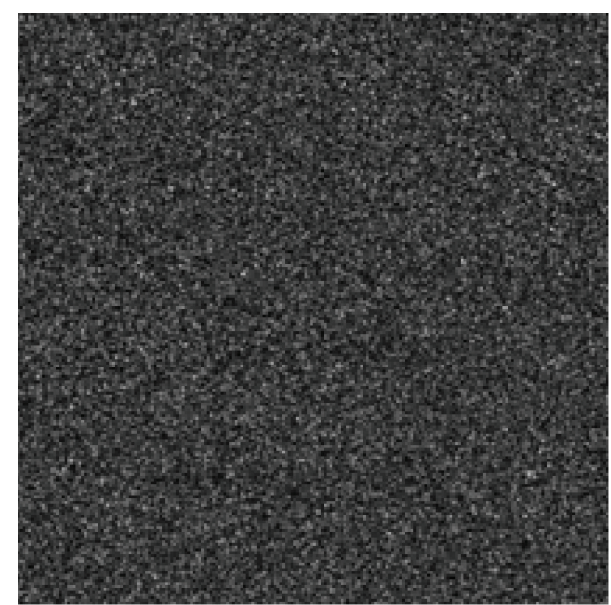

(b)

FIGURE 8: Reconstructed deformed fringe (a) and gray image (b) with wrong phase masks.

Following Fresnel hologram reconstruction and decryption, reconstructed phase and $Z$ coordinate are shown in Figures 7(a) and 7(b). For specific rows (e.g., 80th) of the reconstruction, the comparison between original and reconstructed phase and $Z$ coordinate is shown in Figures $7(\mathrm{c})$ and $7(\mathrm{~d})$, where the mean square errors (MSEs) are 0.0068 and 0.0012 .

With wrong phase masks, both deformed fringe and gray image could not be decrypted as illustrated in Figure 8.
Results of deformed fringe and gray image showed the reduced phase and gray image quality with increased occlusion attack ratio shown in Figure 9.

The mean square error (MSE) and correlation coefficients (CC) used to evaluate the decrypted quality between the reconstructed images without occlusion attacks and the reconstructed images under different occlusion attacks are displayed in Figures 10(a) and 10(b). In Figure 10(a), the blue line presents the MSE between reconstructed $Z$ coordinates 


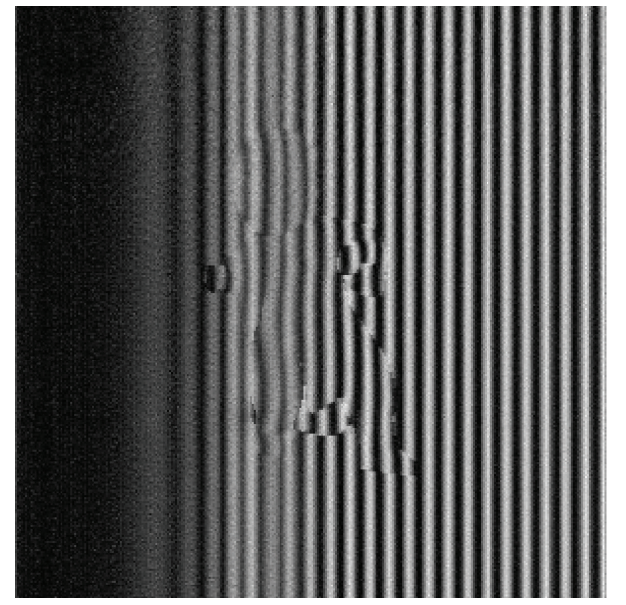

(a)

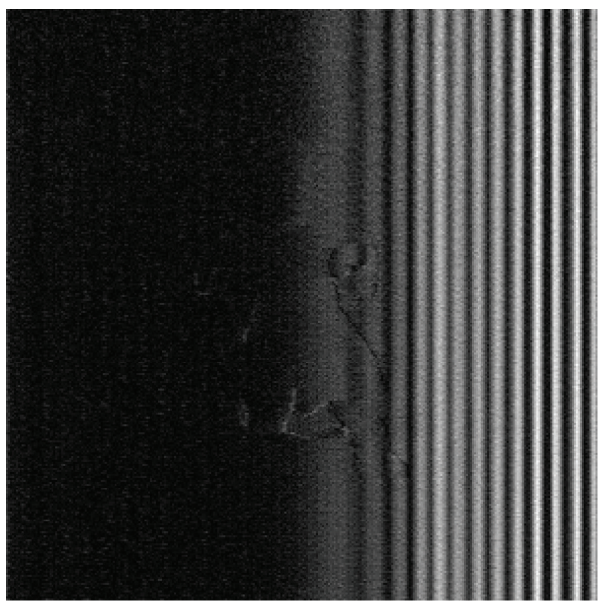

(c)

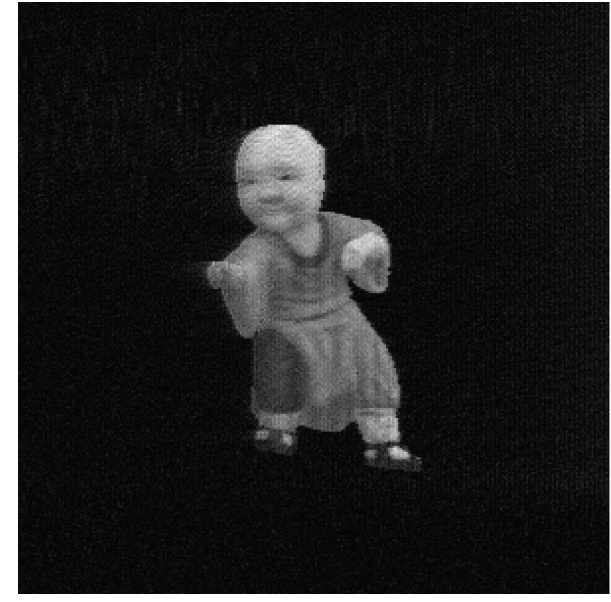

(b)

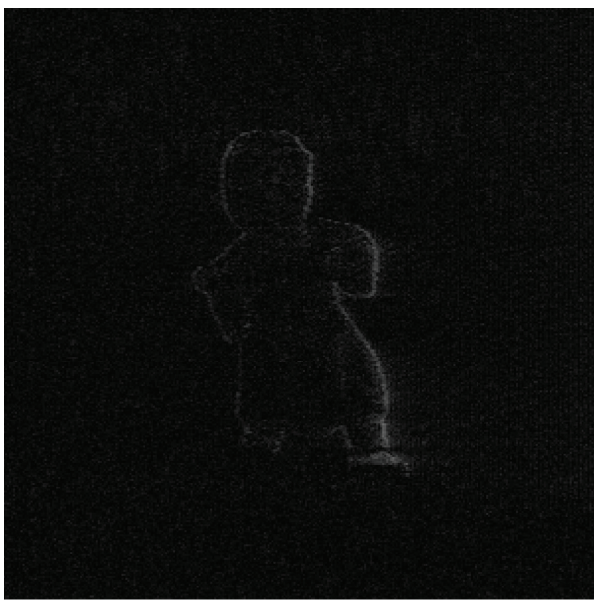

(d)

FiguRE 9: Reconstructed deformed fringe and gray image with occlusion attack ratio of $40 \%$ ((a) and (b)) and 60\% ((c) and (d)).

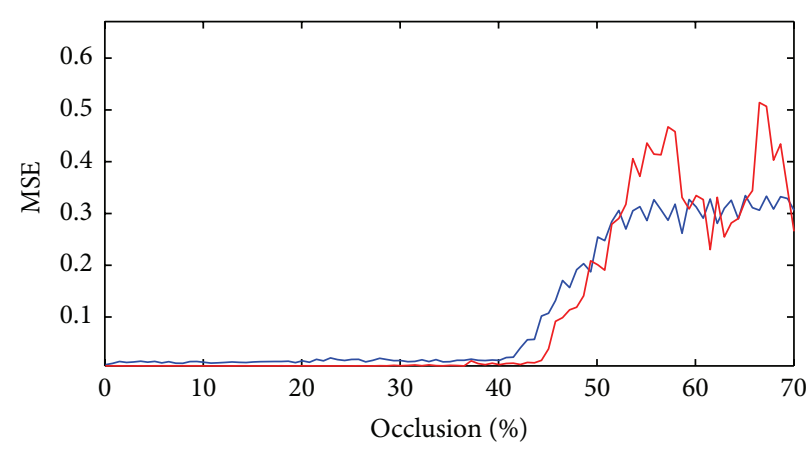

- Recovered $Z$ with directly encrypting $z$

- Recovered $Z$ with phase coding

(a)

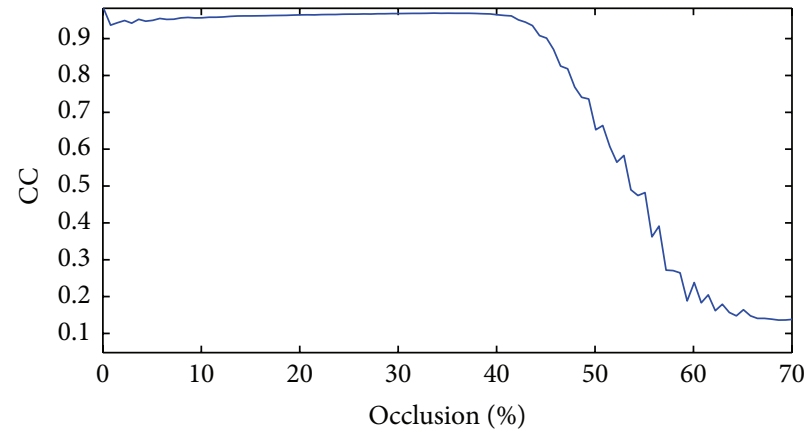

(b)

FIGURE 10: Mean square error (MSE) values of reconstructed $Z$ coordinate for different occlusion attacks (a). Correlation coefficients (CC) values for reconstructed gray image under different occlusion attacks (b). 
without occlusion and with different occlusion attacks when $z$ coordinate is directly used for encryption. And the red line presents the MSE when the phase coding method is used. The phase coding method can reduce the reconstructed $Z$ coordinate error compared with the directly encrypted $z$ coordinate under $50 \%$ occlusion attacks. Above $50 \%$ attacks, the comparison is meaningless because $Z$ coordinate cannot be calculated from both methods.

\section{Conclusion}

In this research, a 3D point cloud object encryption method was proposed. The method uses off-axis digital Fresnel hologram and phase masks for encrypting and decrypting 3D information of the object, in contrast to conventional digital hologram encryption method, which has been restricted to encryption and decryption of $2 \mathrm{D}$ or $3 \mathrm{D}$ objects with Fresnel hologram. The mapping relationship between $X, Y$, and $Z$ coordinates was formulated and, for $Z$ coordinate, a transformation was conducted to translate it to deformed fringe. With deformed fringe, Fresnel hologram encryption and decryption were performed.

Results showed that the proposed method is able to encrypt and decrypt the deformed fringe and gray image of the $3 \mathrm{D}$ object. The proposed method is also sufficiently accurate just yielding small errors between reconstructed $Z$ coordinate and real $Z$ coordinate as shown in Figure $7(d)$. The method is also robust for different occlusion attacks as shown in Figure 9. Additionally, in comparison to direct $Z$ coordinate encryption, deformed fringe is associated with smaller MSE for the occlusion attack ratio under $50 \%$ as illustrated in Figure 10. It indicates that the deformed fringe is more robust than directly using $Z$ coordinate for encryption. This might be because deformed fringe is less sensitive to increased noise-signal ratio relative to simply using $Z$ coordinate.

One possible contribution of this research is that, by transforming the $Z$ coordinate of $3 \mathrm{D}$ object to deformed fringe, it increases the encryption strength because without knowing the mapping relationship between deformed fringe and $Z$ coordinate, $Z$ coordinate decryption and reconstruction would be challenging.

One major limitation of the research should be noted. This study has only investigated $Z$ coordinate deformed fringe encryption and decryption. It would be ideal if all $X, Y, Z$, and gray image of the $3 \mathrm{D}$ object can be transformed, encoded, and encrypted, which can further increase the encryption strength. This however could be also an interesting topic to study in the future.

\section{Conflict of Interests}

The authors Xin Yang and Hongbo Zhang declare that there is no conflict of interests regarding the publication of this paper.

\section{Acknowledgments}

This work has been supported by the National Natural Science Foundation of China under Grant no. 11374267. Xin
Yang thanks Professor Hui Wang and Ting-Chung Poon for their support and encouragement.

\section{References}

[1] X.-W. Li, S.-J. Cho, I.-K. Lee, and S.-T. Kim, “Three-dimensional image security system combines the use of smart mapping algorithm and fibonacci transformation technique," Journal of Applied Research and Technology, vol. 12, no. 6, pp. 1092-1102, 2014.

[2] L. Sui, H. Lu, Z. Wang, and Q. Sun, "Double-image encryption using discrete fractional random transform and logistic maps," Optics and Lasers in Engineering, vol. 56, pp. 1-12, 2014.

[3] L. Krikor, S. Baba, T. Arif, and Z. Shaaban, "Image encryption using DCT and stream cipher," European Journal of Scientific Research, vol. 32, no. 1, pp. 47-57, 2009.

[4] L. Chen and D. Zhao, "Optical color image encryption by wavelength multiplexing and lensless Fresnel transform holograms," Optics Express, vol. 14, no. 19, pp. 8552-8560, 2006.

[5] G. Situ and J. Zhang, "Multiple-image encryption by wavelength multiplexing," Optics Letters, vol. 30, no. 11, pp. 1306-1308, 2005.

[6] X. F. Meng, L. Z. Cai, X. F. Xu et al., "Two-step phase-shifting interferometry and its application in image encryption," Optics Letters, vol. 31, no. 10, pp. 1414-1416, 2006.

[7] J. Liu, H. M. Jin, L. H. Ma, Y. Li, and W. M. Jin, "Optical color image encryption based on computer generated hologram and chaotic theory," Optics Communications, vol. 307, pp. 76-79, 2013.

[8] T. Nomura and B. Javidi, "Optical encryption using a joint transform correlator architecture," Optical Engineering, vol. 39, no. 8, pp. 2031-2035, 2000.

[9] S. Soontaranon and J. Widjaja, "Holographic image encryption by using random phase modulation of plane wave," Optics and Lasers in Engineering, vol. 48, no. 10, pp. 994-999, 2010.

[10] A. Nelleri, J. Joseph, and K. Singh, "Digital Fresnel field encryption for three-dimensional information security," Optical Engineering, vol. 46, no. 4, Article ID 045801, 2007.

[11] E. Tajahuerce and B. Javidi, "Encrypting three-dimensional information with digital holography," Applied Optics, vol. 39, no. 35, pp. 6595-6601, 2000.

[12] H. Kim, D.-H. Kim, and Y. H. Lee, "Encryption of digital hologram of 3D object by virtual optics," Optics Express, vol. 12, no. 20, pp. 4912-4921, 2004.

[13] Z. Zhang, G. Liu, Y. Gao, X. Hu, and S. Wang, "A 3-D encryption technique using computer-generated hologram," in Proceedings of the International Conference on Embedded Software and Systems Symposia (ICESS Symposia '08), vol. 41, pp. 3-6, Sichuan, China, July 2008.

[14] C. Weller, R. Kleer, and F. T. Piller, "Economic implications of 3D printing: market structure models in light of additive manufacturing revisited," International Journal of Production Economics, vol. 164, pp. 43-56, 2015.

[15] J. Wang, J. Coburn, C.-P. Liang et al., "Three-dimensional printing of tissue phantoms for biophotonic imaging," Optics Letters, vol. 39, no. 10, pp. 3010-3013, 2014.

[16] X. W. Li, S. J. Cho, and S. T. Kim, "A 3D image encryption technique using computer-generated integral imaging and cellular automata transform," Optik, vol. 125, no. 13, pp. 2983-2990, 2014.

[17] M. Takeda and K. Mutoh, "Fourier transform profilometry for the automatic measurement of 3-D object shapes," Applied Optics, vol. 22, no. 24, pp. 3977-3982, 1983. 
[18] J.-H. Park, M.-S. Kim, G. Baasantseren, and N. Kim, "Fresnel and Fourier hologram generation using orthographic projection images," Optics Express, vol. 17, no. 8, pp. 6320-6334, 2009.

[19] X. Mao, W. Chen, and X. Su, "Improved Fourier-transform profilometry," Applied Optics, vol. 46, no. 5, pp. 664-668, 2007.

[20] N. Singh and A. Sinha, "Optical image encryption using fractional Fourier transform and chaos," Optics and Lasers in Engineering, vol. 46, no. 2, pp. 117-123, 2008.

[21] J. Lang, R. Tao, and Y. Wang, "Image encryption based on the multiple-parameter discrete fractional Fourier transform and chaos function," Optics Communications, vol. 283, no. 10, pp. 2092-2096, 2010. 


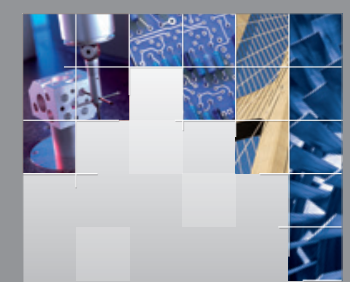

\section{Enfincering}
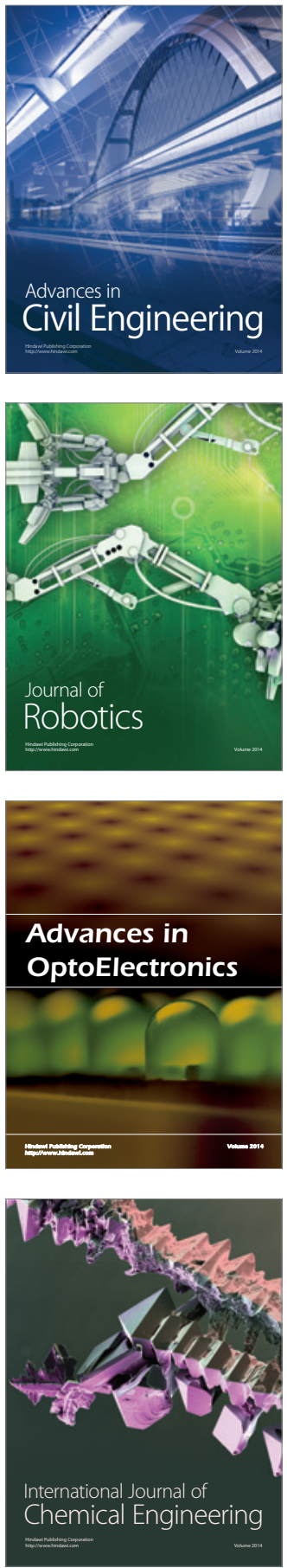

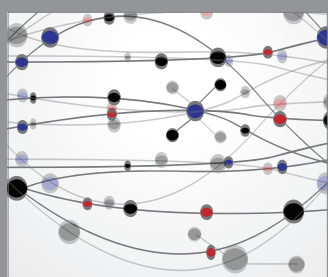

The Scientific World Journal

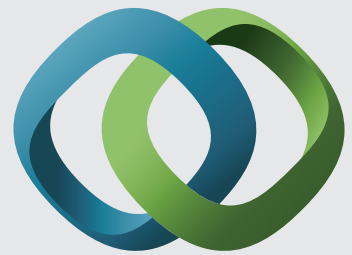

\section{Hindawi}

Submit your manuscripts at

http://www.hindawi.com
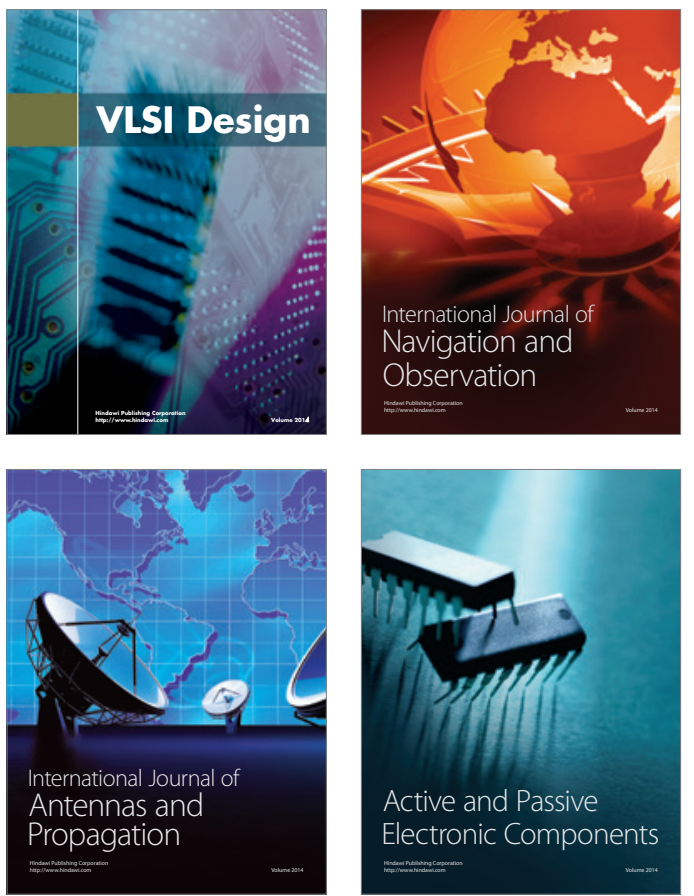
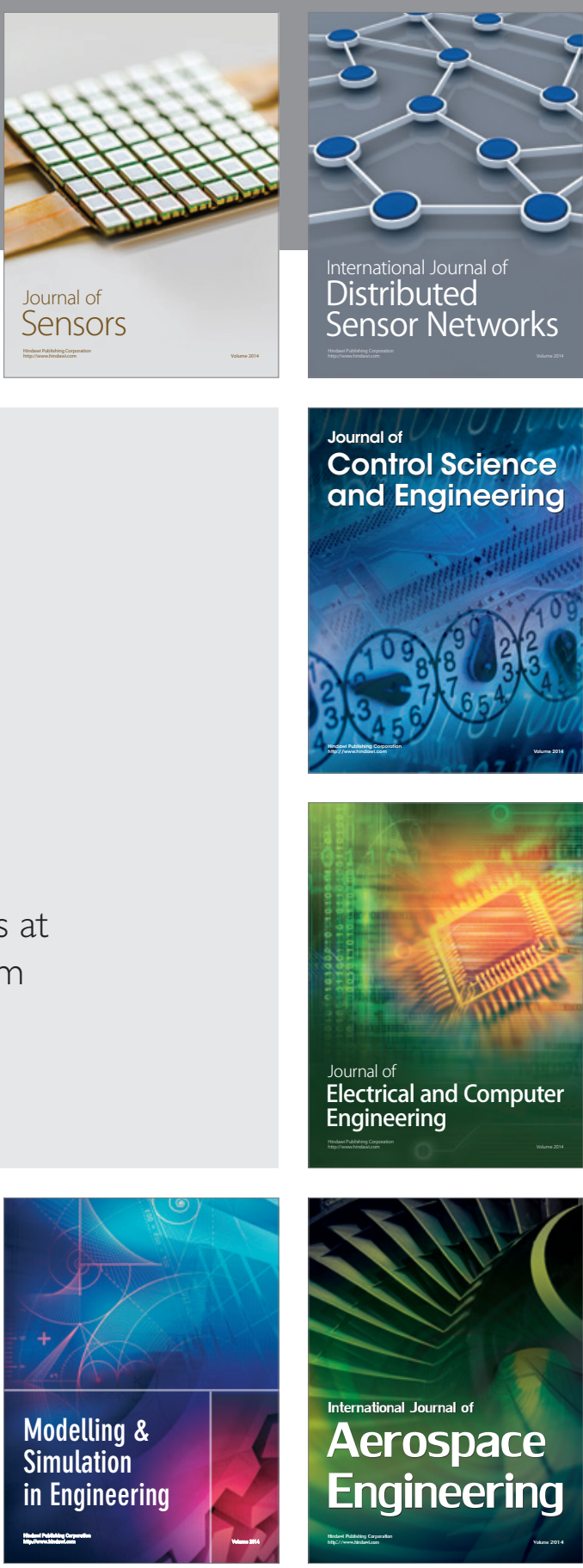

International Journal of

Distributed

Sensor Networks

Journal of

Control Science

and Engineering
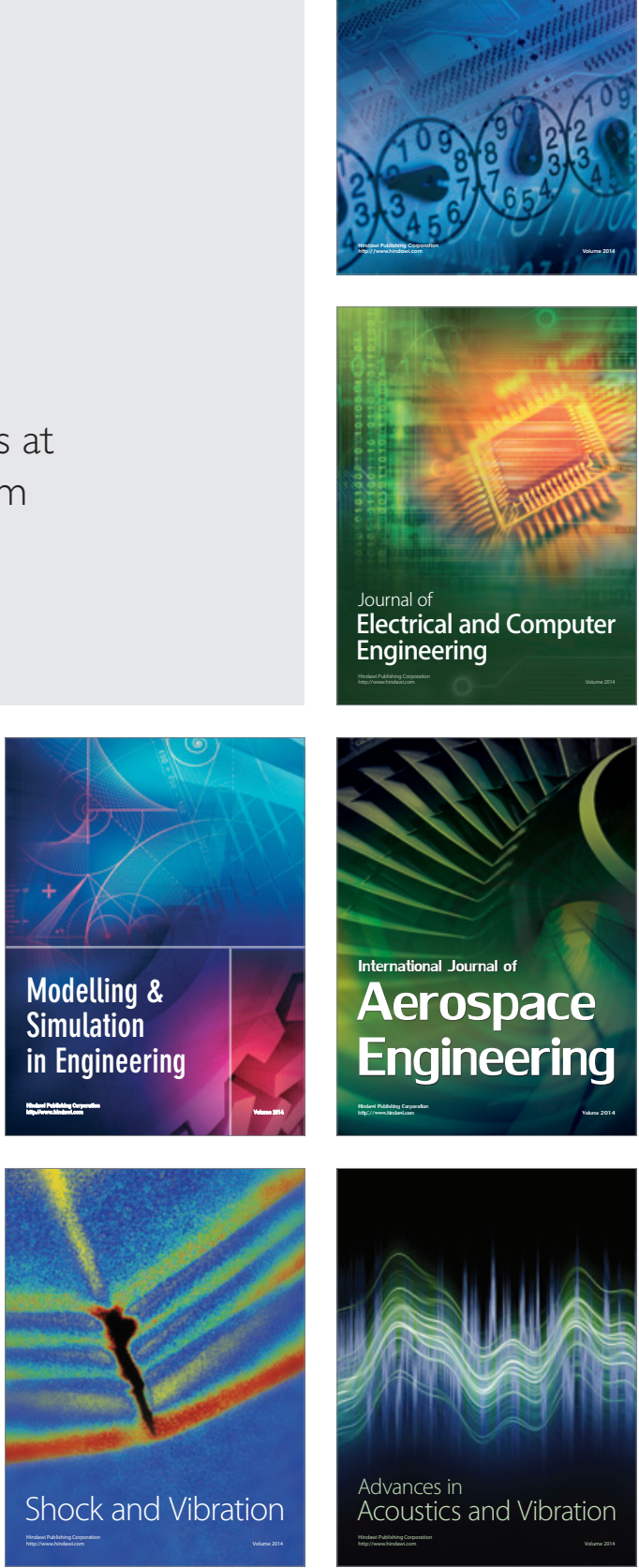